Research Article

\title{
Patient Satisfaction and Associated Factors among Outpatient Health Service Users at Primary Hospitals of North Gondar, Northwest Ethiopia, 2016
}

\author{
Gojjam Eshetie, ${ }^{1}$ Amsalu Feleke, ${ }^{2}$ and Muluken Genetu ${ }^{3}{ }^{3}$ \\ ${ }^{1}$ Department of Medical Laboratory, Denbya Primary Hospital, Gondar, Ethiopia \\ ${ }^{2}$ Department of Health Service Management, University of Gondar, Gondar, Ethiopia \\ ${ }^{3}$ School of Public Health, Wollo University, Dessie, Ethiopia \\ Correspondence should be addressed to Muluken Genetu; mlkngnt@gmail.com
}

Received 13 March 2020; Revised 7 June 2020; Accepted 6 August 2020; Published 24 August 2020

Academic Editor: Guang-Hui Dong

Copyright $(92020$ Gojjam Eshetie et al. This is an open access article distributed under the Creative Commons Attribution License, which permits unrestricted use, distribution, and reproduction in any medium, provided the original work is properly cited.

Introduction. Patient satisfaction with seeking health services is considered as one of the necessary outcomes of health system and measures of health service quality which is directly linked with utilization of the services. The results of this study were crucial and identified important findings for intervention by decision makers on critical need for patient satisfaction improvement as well as to provide evidence for stakeholders in improving quality of outpatient services. This study was aimed at assessing patient satisfaction and associated factors among outpatient health service users at primary hospitals of North Gondar, Northwest Ethiopia. Methods. Facility based cross-sectional study design was conducted in North Gondar from February to March, 2016, among outpatient health service users. Systematic sampling technique was used to get a total of 413 samples. A pretested structured interviewer administered questionnaire was used for data collection. The data were entered to Epi Info version 3.5.1 and exported to SPSS version 20 for analysis. Bivariable and multivariable logistic regression analysis were used to control cofounders and variables with $p$-value less than 0.05 at $95 \% \mathrm{CI}$ were considered as significant. Result. This study showed that the overall patient satisfaction was found to be $56.1 \%$ at $95 \%$ CI (51.0-61.3). Out of all respondents, 218 (53.45\%) were males and 130 (31.9\%) of respondents were in the age group of $\geq 45$ years. Availability of drugs within the hospitals, patient waiting time at registration room, waiting time to see a doctor after registration, and consulting on treatment options were found to be significantly associated with patient satisfaction. Conclusion. The overall patient satisfaction at North Gondar primary hospitals was rated low as compared to national figures. Hospital management bodies and health care service providers should give attention to improvement of drugs availability and reducing waiting time at registration room and the time length to see a doctor after registration in order to improve patients' satisfaction.

\section{Introduction}

Patient satisfaction is the level of service satisfaction that patients experience from having used a health service from a health facility. The quality assurance and accreditation process in most countries requires that the service satisfaction of patients be measured on a regular basis [1]. As a result patient feedback is important to identify problems from health services that need to be resolved in improving patient satisfaction [2].
Patient satisfaction with seeking health services is considered as one of the necessary outcomes of health systems and a measure of health service quality which is directly linked with utilization of the services. Asking patients for what they think about the health service and treatment they have received is an important step for improving the quality of service and for assuring that local health service deliveries are meeting patients' service needs [3]. Therefore, patient satisfaction with a service is a primary indicator of measuring the effectiveness of health care delivery [4]. 
According to World Health Organization (WHO) 2000 report, health systems have to be responsive to the health needs of the patient and the community. The major beneficiaries of a good healthcare system are clearly patients. Actually, it is difficult to measure patients' satisfaction and responsiveness of health systems directly. It importantly depends on the clinical outcomes of patients from health care treatment received [5].

The Ethiopian Federal Ministry of Health (FMOH) has been implementing health sector development program (HSDP). It is a 20 -year plan which was encouraging improvements in the coverage and utilization of the services over the periods of implementation of the health sector development program (HSDP). But, there was still a gap in access and quality of services provided to communities [6].

Studies have been done on this area and pointed out that the levels of patients' satisfaction with health services in different countries of the world vary. A study conducted on patients' satisfaction with services obtained from a Tertiary Care Hospital in Rural Haryana reported $89.1 \%$ patient satisfaction level. However, $20.7 \%$ and $13.0 \%$ of the patients were dissatisfied with the organization's care and cost of care provided, respectively [7].

Evaluation of patients' satisfaction with quality of care provided at the National Health Insurance Scheme clinic of a tertiary hospital in south-eastern Nigeria indicated $66.8 \%$ patient satisfaction level. But patients who were dissatisfied with the hospital's bureaucracy were $48.8 \%$ and with patient waiting time were $48.3 \%$ [8].

In Ethiopia, various studies showed that the levels of patients' satisfaction with health services were found different in different studies. Studies conducted on outpatient services patient satisfaction in Gondar and Jimma hospitals showed $22 \%$ and $57 \%$ satisfaction levels, respectively $[9,10]$. Another study conducted at Jimma specialized hospital indicated $77 \%$ overall client satisfaction level [11].

Even if different strategies are undertaken to improve the quality of health care service delivery, there is high level of patients' dissatisfaction estimates made in Ethiopia (ranging from $22 \%$ to $77 \%$ ). That was why the main aim of conducting this study was to determine the level of patients' satisfaction at outpatient health services delivery level and identify factors significant with patient satisfaction in North Gondar zonal administration.

Therefore, this study provided evidence-based results to improve health service delivery and help to fill gaps which ultimately contribute to enhancing patient satisfaction within the primary hospitals in North Gondar.

\section{Materials and Methods}

2.1. Study Design and Setting. A facility based cross-sectional study design was conducted at primary hospitals of North Gondar, from February 29 to March 28, 2016.

There were six primary hospitals in North Gondar with a catchment population size of about 3.1 million people. According to the data obtained from the hospitals, the annual cases seen as outpatient service users at an average level were 42,354 [12].
2.2. Population. All patients visiting hospitals for seeking outpatient department (OPD) health services were source population of the study while all patients visiting OPDs to get health care services during the data collection period were the study population.

2.3. Eligibility Criteria. All patients of all ages (for children with their attendants) who were presented to OPDs to get health services were included in the study, but from these, patients who were in the condition of very seriously ill and with no attendants and unable to communicate were excluded from the study.

\subsection{Sample Size Determination and Sampling Procedures.} By considering the following assumptions, the sample size $(n)$ of this study was determined using single population formula. A proportion of patient satisfaction of $57.7 \%$ [4], expected margin of error (d) $5 \%$ at $95 \%$ confidence interval, and $10 \%$ nonresponse rate were used.

$$
\text { Thus } \begin{aligned}
n & =\left(Z_{\alpha / 2}\right)^{2} \times \frac{P(1-P)}{d^{2}}, \\
& n=(1.96)^{2} \times \frac{(0.577)(0.423)}{(0.05)^{2}}=375, \\
n & =375+(0.1 \times 375)=413 \text { samples. }
\end{aligned}
$$

Using Epi Info version 7 double population proportion formula for three factors from previous studies, sample sizes were determined but the results were less than the above sample size, and the final sample size of this study used for data collection was 413 .

Samples were accessed for data collection $n$ after the total sample size was proportionally allocated to each primary hospital; based on the average monthly outpatient flow to their internal medicine outpatient department, we took the last year the same month outpatient flow from the log book at each OPD as the baseline for estimating OPDs patient flow. Then, systematic sampling was used to access each participant from each primary hospital. $K$-value was taken as 9 and the samples for each hospital were proportionally allocated systematic sampling at $K$-value 9 , which was applied for all hospitals (Figure 1).

2.5. Study Variables. Level of patient satisfaction (yes/no) was the dependent variable of the study. Independent variables of this study were the following: sociodemographic factors: age, sex, marital status, educational status, occupation, residence, payment status (free/paying), etc.; communication with doctors/nurse: respect and good behavior, getting privacy, being understandable, punctuality, and perceived capacity; organization factors: physical environment: condition of service delivery room, availability of sign and direction indicators, access to water, latrine, and hand washing facility; medication provision: description of side effects of drug, pain control, and availability of drugs; waiting time: registration services, time to receive drug, and 


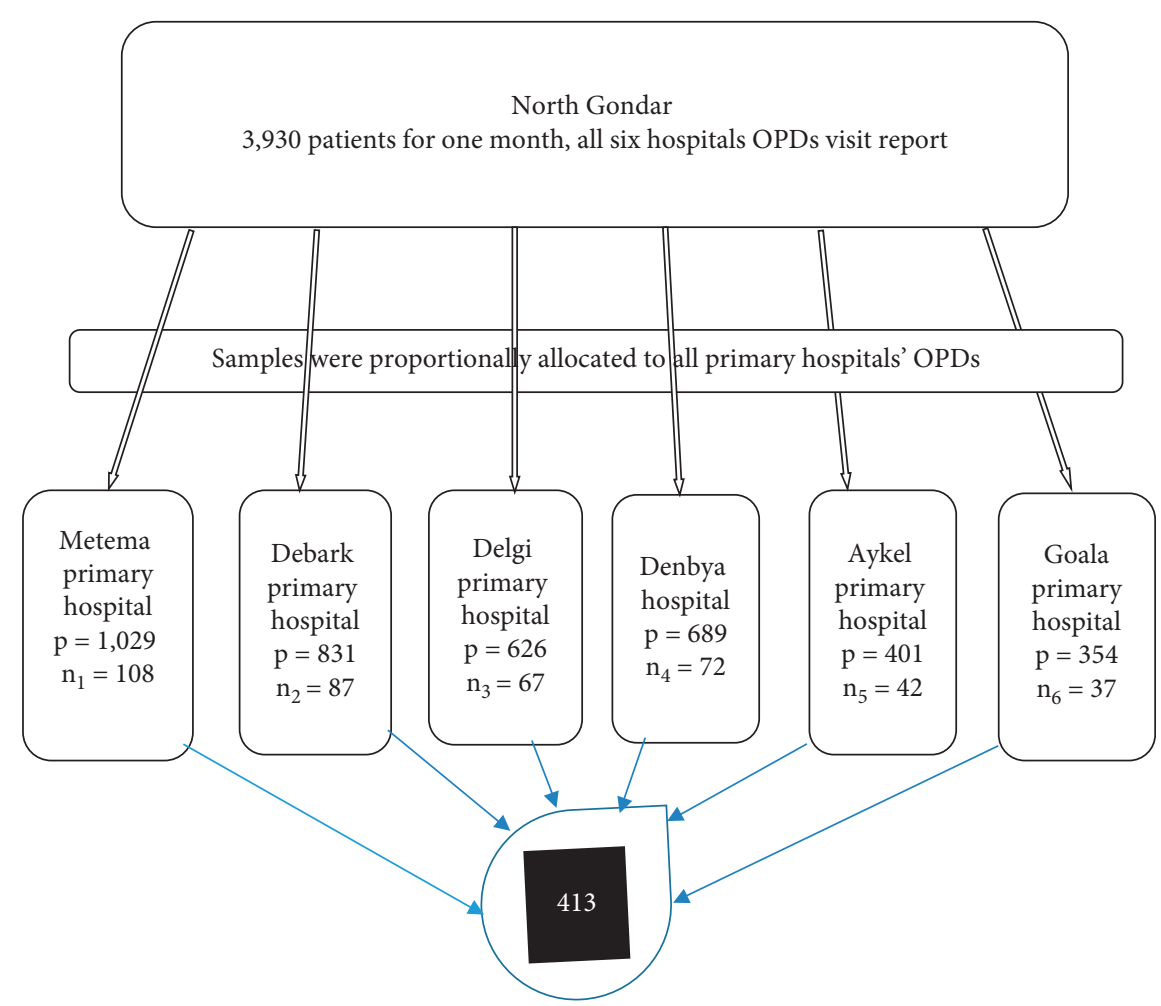

FIGURE 1: Schematic representation of sampling procedures for patient satisfaction and associated factors at primary hospitals of North Gondar, Ethiopia.

time to see a doctor; and convenience of service $[3,4,7,9,13]$.

2.6. Data Collection Procedures, Quality Assurance, and Data Analysis. Patient satisfaction was measured by using the four components of patient satisfaction (acknowledgement, support, accept, and prepare to act (ASAP)) (WHO, 2006); each one of the components were measured with items (4 items for acknowledgement, support, and accept; 3 items for prepare to act). Scores for each component were computed out of 100 and then categorized as "satisfied" for the computed results of $75 \%$ and above; otherwise, scores were categorized as "dissatisfied" for each of the first three components of patient satisfaction; and $67 \%$ and above for the fourth component was used as "satisfied." Then the overall patient satisfaction considered patients who were satisfied with all the components $[14,15]$.

Data was collected using pretested structured interviewer administered questionnaire through exit interview. The questionnaire was adapted from related literatures with slight modification made with the objective of this particular study. Before the actual data collection, two B.S. nurse data collectors were recruited. Training was given on the basic techniques of interviewing to facilitate data collection. They practiced pretesting of the questionnaire after their training. Pretesting of the questionnaire was made before the actual data collection to ensure the acceptability and understandability by the participants at
Gondar specialized hospital. Based on the result, no modification was made.

The English questionnaires were translated to Amharic (local language) for ease of communication during interview. The questionnaire was pretested prior to the actual data collection and appropriate minor modification was made. Regular supervision and reviewing of the completed questionnaire were carried out daily by the principal investigator.

Data were checked, cleaned for completeness and accuracy, entered into Epi Info version 3.5.1, and then exported and analyzed using SPSS version 20.

Descriptive statistics was used for the study variables to describe most of the findings. Bivariate logistic regression analysis was done and variables with $p$-value less than 0.25 were included in multivariate logistic regressions analysis to control confounder and identify the most significant predictor of outcome variables.

2.7. Ethical Considerations. The ethical approval and clearance for the study before data collection were obtained from University of Gondar. Written consent and assent was provided to respondents according to their age. To ensure privacy and confidentiality, the exit interview was conducted where questions and answers cannot be overheard (separate rooms prepared). The participants were also informed that the information obtained from them would not be disclosed to the third person/body. Name and other identifying information was not used in the data collection. 


\section{Result}

3.1. Sociodemographic Characteristics of the Respondents. Among the total sample size (413), four hundred and eight patients were exit-interviewed, giving a response rate of $98.8 \%$. The age range of respondents was from 3 to 80 years with the mean age of 36.3 years. Out of the study subjects, $53.4 \%$ were males. Majority of the respondents, $364(89.2 \%)$, were Orthodox in religion. Majority of the study participants $(47.5 \%)$ were married. Majority of the respondents, 346 $(84.8 \%)$, were paying for health services (Table 1 ).

\subsection{Pattern of Organizational Factors and Communication} with Doctors/Nurses. Among the total respondents, 208 (51\%) respondents were satisfied by communication with their doctors while 220 (53.9\%) were dissatisfied by communication with nurses. Majority of the respondents, 233 (57.1\%), were dissatisfied with physical environment of the hospitals and 254 (62.3\%) were dissatisfied with medication provision. Two hundred and forty-two (59.3\%) of the respondents were reported to have got all the prescribed drugs from the hospital pharmacy. Majority of the respondents (55.9\%) were registered timely and 221 (54.2\%) waited 1-2 hours to see a doctor after registration (Table 2).

3.3. Determining Patient Satisfaction. Overall patient satisfaction with outpatient health care services delivery in this study was $56.1 \%$ (95\% CI: 51-61.3\%) computed from components of patient satisfaction measuring items (Figure 2).

3.4. Factors Associated with Patient Satisfaction in North Gondar Primary Hospitals. In binary logistic regression, eight variables were identified at $p$-value less than 0.25 to be fitted for multivariable logistics regression. These variables were marital status, communication with doctor/nurse, physical environment, medication provision, drug availability, waiting time at registration and waiting time to see a doctor, and convenience.

In multivariable logistic regression, availability of drug (AOR: 0.15, 95\% CI: 0.061-0.365), waiting time at registration process (AOR: 0.052, 95\% CI: 0.023-0.116), waiting time to see a doctor after registration of 1-2 hours (AOR: 0.266, 95\% CI: 0.075-0.942), and greater than two hours (AOR: 0.015 , 95\% CI: $0.003-0.091$ ) were statically significantly associated with patient satisfaction.

Those patients who have got some of the prescribed drugs reduced the odds of patient satisfaction by $85 \%$ when compared to patients who have got all drugs (AOR: 0.15, 95\% CI: 0.061-0.365). Those patients whose registration process was not done timely reduced the odds of patient satisfaction by $95 \%$ when compared to patients who registered timely (AOR: 0.052, 95\% CI: 0.023-0.116). Those patients waiting 1-2 hours to see a doctor reduced the odds of patient satisfaction by $73 \%$ when compared to patients waiting less than one hour (AOR: 0.266, 95\% CI: 0.075-0.942). In addition, those patients waiting time greater than two hours to see a doctor reduced the odds of patient satisfaction by $98 \%$ when compared to patients waiting less than one hour to see a doctor (AOR: 0.015, 95\% CI: 0.003-0.091) (Table 3).

\section{Discussion}

This study has revealed that the satisfaction level with the outpatient services in North Gondar primary hospitals was low (56.1\%) when compared to the Ethiopian FMOH 2013/ 2014 annual performance report of patient satisfaction (77\%); to the report of the studies conducted in Jimma specialized hospital (77\%), Turkey (70\%), and Trinidad and Tobago (74\%) $[11,16,17]$, respectively. On the other hand, this study showed higher level of satisfaction than study conducted at Tigray zonal hospitals (43.6\%) [18]. The difference might be related to the difference in methods and scopes of the study. This study has shown that lack of medication provision was the major problem affecting the satisfaction status of patients among $62.3 \%$.

On the other hand, the difference might be that specialized hospitals (where those studies were conducted) are well equipped and have sufficient diversity of health workers, better diagnostic facilities, health care infrastructures, and good awareness of health service providers at different levels who are expected to practice the standard ways of patient examination resulting in higher overall satisfaction levels.

This finding is similar to other findings conducted in Jimma hospital (57.1\%), Wolaita Sodo University Teaching Hospital (54.2\%), and hospitals of Eastern Ethiopia (54.1\%) although the proportions in the latter studies were higher where $70 \%, 67 \%$, and $73.4 \%$ of patients did not get all prescribed drugs from primary hospital pharmacies $[10,14,19]$, respectively. The reason for this difference might be due to higher user demand in the latter ones.

Generally, as stated in different studies above and other studies, lack of prescribed drugs was the main reason for dissatisfaction of patients and leads many poor people to bypass the closest public facilities to go to costlier private facilities (World Bank, 2012). This is similarly indicated in this study both in qualitative and in quantitative results.

Factors related to patient satisfaction were prescribed drugs availability, waiting time to see a doctor, and consultation on treatment options, which were significantly associated with patient satisfaction and were the important predictor variables found in this study.

The study has shown that failure of getting all prescribed drugs was negatively associated with patient satisfaction. Similar finding was found on a study conducted in Wolaita Sodo University Teaching Hospital, in Mettu rural areas, and in hospitals of Amhara region [13, 14, 20]. In this study, $59.3 \%$ of the patients did get all prescribed drugs from the hospitals' pharmacies. This finding is comparable to that of the study conducted in Wolaita Sodo University Teaching Hospital and in hospitals of Amhara region [13, 14] where $64.3 \%$ and about two-thirds of total patients did get all prescribed drugs from the hospital pharmacy, respectively. In other studies conducted in Jimma University Specialized Hospital, in Jimma hospital, and in Tigray zonal hospitals 
TABLE 1: Sociodemographic characteristics of the respondents in North Gondar primary hospitals, $2016(n=408)$.

\begin{tabular}{|c|c|c|c|}
\hline Variables & Category & Frequency $(\mathrm{n})$ & Percentage (\%) \\
\hline \multirow{2}{*}{ Sex } & Male & 218 & 53.4 \\
\hline & Female & 190 & 46.6 \\
\hline \multirow{5}{*}{ Age } & $<15$ years & 26 & 6.4 \\
\hline & $15-24$ years & 77 & 18.9 \\
\hline & $25-34$ years & 101 & 24.8 \\
\hline & $35-44$ years & 74 & 18.1 \\
\hline & $\geq 45$ years & 130 & 31.9 \\
\hline \multirow{3}{*}{ Religion } & Orthodox & 364 & 89.2 \\
\hline & Muslim & 39 & 9.6 \\
\hline & Others & 5 & 1.2 \\
\hline \multirow{3}{*}{ Ethnicity } & Amhara & 389 & 95.3 \\
\hline & Tigray & 14 & 3.5 \\
\hline & Kimant & 5 & 1.2 \\
\hline \multirow{4}{*}{ Marital status } & Single & 151 & 37 \\
\hline & Married & 194 & 47.5 \\
\hline & Divorced & 37 & 9.1 \\
\hline & Widowed & 26 & 6.4 \\
\hline \multirow{4}{*}{ Educational status } & Unable to read and write & 132 & 32.4 \\
\hline & Grades 1-6 & 102 & 25 \\
\hline & Grades 7-12 & 104 & 25.5 \\
\hline & Diploma and above & 70 & 17.2 \\
\hline \multirow{6}{*}{ Occupation } & Government employee & 77 & 18.9 \\
\hline & Merchant & 57 & 14 \\
\hline & Farmer & 146 & 35.8 \\
\hline & No job & 36 & 8.8 \\
\hline & Student & 50 & 12.3 \\
\hline & Others & 42 & 10.3 \\
\hline \multirow{2}{*}{ Residence } & Urban & 205 & 50.2 \\
\hline & Rural & 203 & 49.8 \\
\hline \multirow{2}{*}{ Payment status } & Paying & 346 & 84.8 \\
\hline & Free & 62 & 15.2 \\
\hline \multirow{3}{*}{ Reason for visit } & Illness & 404 & 99 \\
\hline & Family planning & 3 & 0.7 \\
\hline & Others & 1 & 0.2 \\
\hline \multirow{2}{*}{ Frequency of visit } & New & 203 & 49.8 \\
\hline & Repeat & 205 & 50.2 \\
\hline \multirow{4}{*}{ Family monthly income } & $<1200$ birr & 224 & 54.9 \\
\hline & $1200-2200$ birr & 80 & 19.6 \\
\hline & $2201-3200$ birr & 83 & 20.3 \\
\hline & $>3200$ birr & 21 & 5.1 \\
\hline
\end{tabular}

$[10,11,18], 70 \%, 66.7 \%$, and $61 \%$ of the patients did not get all or some of the prescribed drugs from hospitals pharmacies, respectively.

The findings of this study reported that long waiting time to see a doctor after registration was found to be negatively associated with the level of patient satisfaction. There were similar findings in the study of Jimma specialized hospital, Debre Berhan referral hospital, and Eastern Ethiopia $[4,11,19]$. In this study, $25 \%$ of the respondents were dissatisfied with waiting time to see a doctor which is comparable to the study conducted in Bangladesh and in Agra district health facilities [21, 22] where $28.2 \%$ and $29.5 \%$, respectively, of the patients were dissatisfied with long waiting time to see a doctor after registration.
According to the results of the present study consultation on treatment options is the important factor predicting the levels of patient satisfaction. As health service providers continue consulting their patients on treatment options, patient satisfaction increases. So, it indicates that it is better to give attention to outpatient consultation on treatment options to improve patient satisfaction. Similar study results done in Wolaita Sodo University Teaching Hospital support this finding [14].

Moreover, the present study showed that long waiting time in registration process was another negatively associated factor with patient satisfaction which is similar to a study conducted in hospitals of Amhara region and a study conducted in Agra district health facilities which also 
TABLE 2: Patient responses for organizational factors among outpatient health service users in North Gondar, Ethiopia, 2016 ( $n=408$ ).

\begin{tabular}{|c|c|c|c|}
\hline Variables & Category & Frequency $(\mathrm{n})$ & Percentage $(\%)$ \\
\hline \multirow{2}{*}{ Satisfied with communication with doctors } & Yes & 208 & 51 \\
\hline & No & 200 & 49 \\
\hline \multirow{2}{*}{ Satisfied with communication with nurse } & Yes & 188 & 46.1 \\
\hline & No & 220 & 53.9 \\
\hline \multirow{2}{*}{ Satisfied with physical environment } & Yes & 175 & 42.9 \\
\hline & No & 233 & 57.1 \\
\hline \multirow{2}{*}{ Satisfied with medication provision } & Yes & 154 & 37.7 \\
\hline & No & 254 & 62.3 \\
\hline \multirow{3}{*}{ Availability of prescribed drugs } & All & 242 & 59.3 \\
\hline & Some & 136 & 33.3 \\
\hline & None & 30 & 7.4 \\
\hline \multirow{2}{*}{ Registration process was done timely } & Yes & 228 & 55.9 \\
\hline & No & 180 & 44.1 \\
\hline \multirow{3}{*}{ Waiting time to be seen by doctor } & $<1$ hour & 86 & 21.1 \\
\hline & 1-2 hour & 221 & 54.2 \\
\hline & $>2$ hour & 101 & 24.8 \\
\hline \multirow{3}{*}{ Waiting time for receiving drugs } & Long & 69 & 16.9 \\
\hline & Fair & 237 & 58.1 \\
\hline & Short & 102 & 25 \\
\hline \multirow{2}{*}{ Satisfied with overall waiting time } & Yes & 211 & 51.7 \\
\hline & No & 197 & 48.3 \\
\hline \multirow{2}{*}{ Consulting on treatment options } & Yes & 209 & 51.2 \\
\hline & No & 199 & 48.8 \\
\hline
\end{tabular}

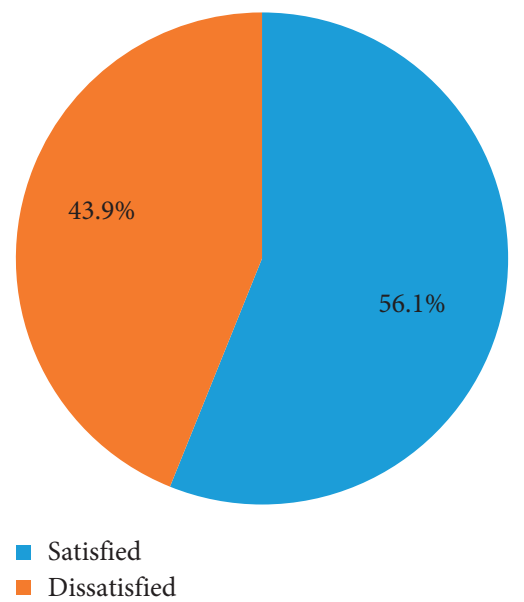

Figure 2: Patient satisfaction for outpatient health service users in North Gondar, Ethiopia, $2016(n=408)$.

TABLE 3: Bivariable and multivariable analysis of factors affecting patient satisfaction in North Gondar, Northwest Ethiopia, 2016 ( $n=408$ ).

\begin{tabular}{|c|c|c|c|c|c|}
\hline \multirow{2}{*}{ Variables } & \multirow{2}{*}{ Category } & \multicolumn{2}{|c|}{ Patient satisfaction } & \multicolumn{2}{|c|}{ OR at $95 \% \mathrm{CI}$} \\
\hline & & Satisfied $n(\%)$ & Dissatisfied $n(\%)$ & $\mathrm{COR}$ & AOR \\
\hline \multirow{4}{*}{ Marital status } & Single & $87(57.6)$ & $64(42.4)$ & 1 & 1 \\
\hline & Married & $113(58.2)$ & $81(41.8)$ & $1.06(0.667-1.579)$ & $0.981(0.439-2.192)$ \\
\hline & Divorced & $14(37.8)$ & $23(62.2)$ & $0.448(0.214-0.9)^{*}$ & $0.346(0.08-1.40)$ \\
\hline & Widowed & $15(57.7)$ & $11(42.3)$ & $1.003(0.43-2.33)$ & $0.379(0.09-1.60)$ \\
\hline \multirow{2}{*}{ Communication with doctors } & Yes & $161(77.4)$ & $47(22.6)$ & 1 & 1 \\
\hline & No & $68(34)$ & $132(66)$ & $0.15(0.09-0.23)^{* *}$ & $0.44(0.17-1.13)$ \\
\hline \multirow{2}{*}{ Communication with nurses } & Yes & $152(80.9)$ & $36(19.1)$ & 1 & 1 \\
\hline & No & $77(35)$ & $143(65)$ & $0.128(0.081-0.201)^{* *}$ & $0.39(0.13-1.22)$ \\
\hline \multirow{2}{*}{ Physical environment } & Yes & $143(81.7)$ & $32(18.3)$ & 1 & 1 \\
\hline & No & $86(36.9)$ & $147(63.1)$ & $0.131(0.082-0.209)^{* *}$ & $1.019(0.389-2.673)$ \\
\hline
\end{tabular}


TABLE 3: Continued.

\begin{tabular}{|c|c|c|c|c|c|}
\hline \multirow{2}{*}{ Variables } & \multirow{2}{*}{ Category } & \multicolumn{2}{|c|}{ Patient satisfaction } & \multicolumn{2}{|c|}{ OR at $95 \% \mathrm{CI}$} \\
\hline & & Satisfied $n(\%)$ & Dissatisfied $n(\%)$ & COR & AOR \\
\hline \multirow{2}{*}{ Provision of medication } & Yes & $125(85.2)$ & $29(18.8)$ & 1 & 1 \\
\hline & No & $104(40.9)$ & $150(59.1)$ & $0.161(0.1-0.259)^{* *}$ & $3.0(1.001-8.987)$ \\
\hline \multirow{3}{*}{ Drug availability } & All & $192(79.3)$ & $50(20.7)$ & 1 & 1 \\
\hline & Some & $34(25)$ & $102(75)$ & $\begin{array}{c}0.087 \\
(0.053-0.143)^{* *}\end{array}$ & $0.15(0.061-0.365)^{* *}$ \\
\hline & None & $3(10)$ & $27(90)$ & $\begin{array}{c}0.029 \\
(0.008-0.099)^{* *}\end{array}$ & $0.148(0.02-1.089)$ \\
\hline \multirow{2}{*}{ Registration process was done timely } & Yes & $199(87.3)$ & $29(12.7)$ & 1 & 1 \\
\hline & No & $30(16.7)$ & $150(83.3)$ & $0.029(0.017-0.051)^{* *}$ & $0.052(0.023-1.116)$ \\
\hline \multirow{3}{*}{$\begin{array}{l}\text { Waiting time to see a doctor after } \\
\text { registration }\end{array}$} & $<1$ hour & $81(94.2)$ & $5(5.8)$ & 1 & 1 \\
\hline & 1-2 hours & $141(63.8)$ & $80(36.2)$ & $0.109(0.042-0.28)^{* *}$ & $0.266(0.075-0.942)^{*}$ \\
\hline & $>2$ hours & $7(6.9)$ & $94(93.1)$ & $0.005(0.001-0.015)^{* *}$ & $\begin{array}{c}0.015 \\
(0.003-0.091)^{* *} \\
\end{array}$ \\
\hline \multirow[b]{2}{*}{ Consulting on treatment options } & Yes & $174(83.3)$ & $35(16.7)$ & 1 & 1 \\
\hline & No & $55(27.6)$ & $144(72.2)$ & $\begin{array}{c}0.077 \\
(0.048-0.124)^{* *}\end{array}$ & $0.502(0.177-0.725)$ \\
\hline
\end{tabular}

${ }^{* *} p<0.001,{ }^{*} p<0.05, \mathrm{COR}=$ crude odds ratio, $\mathrm{AOR}=$ adjusted odds ratio, and $95 \% \mathrm{CI}=95 \%$ confidence interval.

reported that registration procedure was a cause of dissatisfaction $[13,22]$.

\section{Limitation of the Study}

There might be social desirability bias due to the fact that facility based studies produce more positive responses by the patient. This may result in relatively short-lived "halo effect" whereby patients feel more satisfied immediately after their consultation than they do afterwards. It should also be noted that the reliance on the response of parents or caregivers for their children might introduce surrogate bias.

\section{Conclusion}

The overall level of patients' satisfaction towards outpatient health care services in North Gondar primary hospitals was low. Availability of drugs and supplies, waiting time in registration process, and waiting time to see a doctor after registration were significant predictors of patient dissatisfaction. Hospital management bodies and health service providers need to give attention to improvement of drugs availability and reducing waiting time in registration process and time to see a doctor after registration in order to improve level of patient satisfaction. It is necessary to conduct further study and periodic assessment of health services delivery in regard to patient satisfaction.

\section{Abbreviations}

ART: Antiretroviral therapy

B.S.: $\quad$ Bachelor of science

DC: Data collectors

DEC: Data entry clerk

FMOH: Federal Ministry of Health

HSDP: Health sector development program

MPH: Master of public health
OPDs: Outpatient departments

PI: $\quad$ Principal investigator

RA: Research advisors

SPSS: Statistical package for the social science

UoG: University of Gondar

VCT: Volunteer counseling testing

WHO: World Health Organization.

\section{Data Availability}

The data used are available upon reasonable request to the corresponding author.

\section{Ethical Approval}

Ethical clearance and approval letter were obtained from the University of Gondar Ethical Review Board.

\section{Consent}

Written informed consent was provided to participants. Confidentiality of respondent's information was secured.

\section{Conflicts of Interest}

The authors declare that no conflicts of interest exist in the publication of this paper.

\section{Authors' Contributions}

GE has been involved in the conception, design, analysis, interpretation, and report and manuscript writing. $\mathrm{AF}$ and MG were also involved in the design, analysis, interpretation of the data, and manuscript writing. All authors read and approved the final manuscript. 


\section{Acknowledgments}

The authors would like to thank all study participants for their cooperation in providing the necessary information. The authors would also like to thank data collectors and supervisors for the devotion and quality work during data collection, as well as Amhara National Regional State Health Bureau, UoG.

\section{References}

[1] M. Smith and B. Engelbrecht, Guide to Assessing Client Satisfaction at District Hospitals, The Press Gang, Durban, South Africa, 2001.

[2] P. R. Sodani and K. Sharma, "A study on patient satisfaction at a multi super specialty hospital in Delhi," Hospital Topics, vol. 92, no. 1, pp. 1-6, 2014.

[3] A. Bekele, G Taye, Y Mekonnen et al., "Levels of outpatient satisfaction at selected health facilities in six regions of Ethiopia," Ethiopian Journal of Health Development, vol. 22, no. 1, pp. 42-48, 2008.

[4] R. Mezemir, D. Getachew, and M. Gebreslassie, "Patients' satisfaction and its determinants in outpatient department of deberebirhan referral hospital, North Shoa, Ethiopia," International Journal of Economics \& Management Sciences, vol. 3, no. 3, 2014.

[5] World Health Organization (WHO), World Health Report 2000-Health Systems: Improving Performance, World Health Organization, Geneva Switzerland, 2000.

[6] Federal Ministry of Health of Ethiopia, HSDP IV Annual Performance Report, Federal Ministry of Health of Ethiopia, Addis Ababa, Ethiopia, 2006.

[7] S. S. Qadri et al., "An assessment of patients satisfaction with services obtained from a tertiary care hospital in rural Haryana," International Journal of Collaborative Research on Internal Medicine and Public Health, vol. 4, no. 8, 2012.

[8] G. Iloh et al., "Evaluation of patients' satisfaction with quality of care provided at the National Health Insurance Scheme clinic of a tertiary hospital in South- Eastern Nigeria," Nigerian Journal of Clinical Practice, vol. 15, no. 4, 2012.

[9] M. Dagnew and D. Zakus, "Community perception on OPD performance of a teaching hospital in Gondar town, Ethiopia," Ethiopian Medical Journal of Health Development, vol. 35, no. 3, 1997.

[10] L. Oljira and S. Gebre-selassie, "Satisfaction with outpatient health services at Jimma hospital, South West Ethiopia," Ethiopian Medical Journal of Health Development, vol. 15, no. 3, pp. 179-184, 2001.

[11] F. Assefa, A. Mosse, and Y. H. Michael, "Assessment of client satisfaction with health service deliveries at Jimma University Specialized Hospital," Ethiopian Journal of Health Science, vol. 21, no. 2, 2011.

[12] D. Hapital, "Hospital performance annual report," 2015.

[13] G. Mitike, A. Mekonnen, and M. Osman, "Satisfaction on outpatient services in hospitals of the Amhara region," Ethiopian Medical Journal, vol. 40, no. 4, 2002.

[14] G. G. Sagaro, A. W. Yalew, and M. M. Koyira, "Patients' satisfaction and associated factors among outpatient department at Wolaita Sodo university teaching hospital, southern Ethiopia: a cross sectional study," Science Journal of Clinical Medicine, vol. 4, no. 5, pp. 109-116, 2015.

[15] A. Tayelgn, D. T. Zegeye, and Y. Kebede, "Mothers' satisfaction with referral hospital delivery service in Amhara
Region, Ethiopia," BMC Pregnancy and Childbirth, vol. 11, no. 78, 2011.

[16] S. Bodur, Y. E. Zdemir, and F. Kara, "Outpatient satisfaction with health centers in urban areas," Turkish Journal Of Medical Sciences, vol. 32, pp. 409-414, 2002.

[17] H. Singh, E. D. Haqq, and N. Mustapha, "Patients' perception and satisfaction with health care professionals at primary care facilities in Trinidad and Tobago," Bulletin of the World Health Organization, vol. 77, no. 4, 1999.

[18] G. Adane, "Assessment of clients' satisfaction with outpatient services in Tigray Zonal Hospitals," 2006.

[19] B. Abdosh, "The quality of hospital services in eastern Ethiopia: patient's perspective," Ethiopian Journal of Health Development, vol. 20, no. 3, pp. 199-200, 2009.

[20] S. M. Tume, W. B. Salgedo, and F. T. Jaleta, "Patient satisfaction and associated factors with outpatient medical services in rural primary healthcare facilities, Ilubabor zone, Oromiya region, South West Ethiopia," International Journal of Current Research, vol. 4, no. 5, pp. 109-116, 2015.

[21] J. M. Aldana, H. Piechulek, and A. Al-Sabir, "Client satisfaction and quality of health care in rural Bangladesh," Bulletin of the World Health Organization, vol. 79, no. 6, 2001.

[22] D. Anand, Sk Kaushal, and G. SC, "A study on status of client satisfaction in patients attending governmental health facilities in Agra District," Indian Journal of Community Health, vol. 24, no. 3, 2012. 